\title{
Polymers Going Rigid, Thick, and Laterally Infinite
}

\author{
Afang Zhang, Junji Sakamoto, and A. Dieter Schlüter*
}

\begin{abstract}
This short review article provides insight into the impact organic chemistry can have on state-of-the-art materials creation. Three cases were selected from the authors' laboratory, the first being 'Suzuki polycondensation', a powerful method with which innovative organic chemistry was successfully transferred to polymer synthesis and which meanwhile has even found technical scale application. The second describes the decoration of linear polymers with a dense layer of regular branch work. Though seemingly a rather esoteric enterprise, these decorations resulted in considerable property changes as compared to other linear polymers and, additionally, led to the discovery of novel properties. Consequently, this area which is commonly referred to as 'dendronized polymers' in a world-wide activity has been developed into a ripe research field, presently under exploration for possible technical application. Finally, an example still very much in its first tumbling steps was selected to give yet another perspective of the role of organic chemistry in a materials-oriented chemistry. It aims at the generation of ultrathin, yet internally strictly defined, sheet-like macromolecules for which a great future is foreseen, e.g. as a 2D platform for the systematic construction of 3D matter.
\end{abstract}

Keywords: Dendronized polymers · Molecular sheets · Nano objects · Polymer synthesis · Suzuki polycondensation

\section{Introduction}

Polymer synthesis has witnessed an enormous development over the past one or two decades. The variety of accessible structures has dramatically increased and the precision with which they can be made has reached levels one could not have dreamed of in the seventies and eighties of the last century. High tolerance to functional groups, proper placement of predetermined end groups, high level of stereo control and predictable chain lengths and chain length distributions are no longer exceptions. Prominent examples include metallocene polymerizations of olefins, ${ }^{[1]}$ controlled radical polymerizations of a large collection of sometimes even complex monomers, ${ }^{[2]}$

${ }^{\star}$ Correspondence: Prof. Dr. A. D. Schlüter ETH Zürich

Department of Materials, $\mathrm{HCl} \mathrm{J} 541$

Wolfgang-Pauli Str. 10

$\mathrm{CH}-8093$ Zürich

Tel.: +4163 36380

Fax: +416331395

E-mail: ads@mat.ethz.ch and polymerizations using supramolecular growth principles. ${ }^{[3]}$ Besides these more method-oriented developments there has also been quite some progress in research aimed at polymers with principally new molecular structures. A rather remarkable case here is polymers decorated with long 'hairs' at each repeat unit, which are commonly referred to as bottlebrushes. ${ }^{[4]}$ The laboratory of polymer chemistry at ETH as well as the labs at the Max-Planck Institute for Polymer Research, Mainz, Germany, and the Freie Universität Berlin, Germany, where this group had been previously active, have contributed several different examples in which organic chemistry played a substantial role.

Three of these examples will be described here; two of them have already reached considerable maturity whereas the third is still rather premature and one does not know for sure whether it will be ultimately successful. Its potential impact however is considerable.

The first case is a methodological development aiming at solving an old problem in polymer synthesis which was insurmountable until organic chemistry had developed the famous Suzuki-Miyaura cross-coupling reaction back in 1981,[5] a reaction which allows aromatic units to be connected directly to one another. Though many polymers with aromatic units had been synthe- sized over the years and found important applications, e.g. for high performance fibers, there was no method available by which aromatic units could be coupled directly via $\mathrm{C}-\mathrm{C}$ bonds and not via ester, amide and imide groups like in aromatic polyesters, polyamides, and polyimides. This direct coupling would convert such aromatic polymers into polyarylenes; fully aromatic, highly rigid, single-stranded polymers for which interesting properties were predicted, ranging from active components in optoelectronic devices all the way to high strength materials with attractive chemical and thermal stability. It was therefore considered an important task to show whether the Suzuki-Miyaura reaction could possibly be added to the arsenal of polymer synthesis' methodology to solve exactly this problem. ${ }^{[6]}$

The second case deals with the perhaps somewhat naive sounding question; "Where are the limits for steric load of a monomer such that it still polymerizes?", which has more importance than one may think at first glance. If monomers carry increasingly spatially demanding substituents, their polymerizable unit will be increasingly more wrapped and shielded by this substituent which, in turn, renders polymerization increasingly more difficult. If propagation could nevertheless be brought about, however, the resulting poly- 


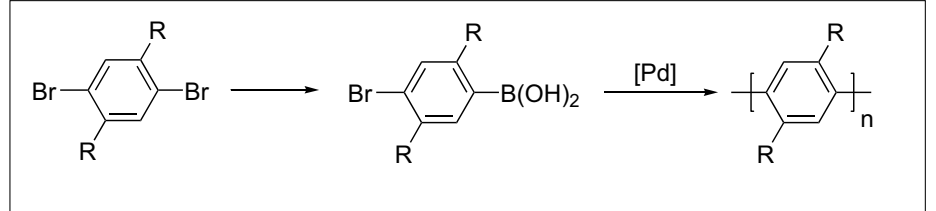

Scheme 1. The first Suzuki polycondensation described in the literature: $[\mathrm{Pd}]=\mathrm{Pd}\left(\mathrm{PPh}_{3}\right)_{4}$ and $\mathrm{R}=$ hexyl

mer backbone would be forced into a more and more stretched conformation which, in the extreme case, could eventually furnish a macromolecule, whose envelope is best described as a nanocylinder. Nanocylinders were considered very attractive research targets some 15 years back because they could serve numerous unprecedented functions which include single-molecule glasses, entities for controlled surface coverage and single-molecule chemistry, counterparts for supramolecular assembly with biomacromolecules, and alike. This field, which - following one of the author's (ADS) suggestion - is meanwhile internationally referred to as 'dendronized polymers', [7] has led to numerous exciting findings and our laboratory is proud of having been part of all this.

The third case is a long-term project aimed at a class of polymers which could not be made so far, though numerous attempts have been published over the years and organic chemists, polymer and materials chemists, physicists and surface scientists have always enthused about, namely: two-dimensional polymers. Practically all bio- and man-made polymers known today consist of single-stranded, covalent backbones. The indispensable functions many of them have both for life as such and in the daily life of every human being result from their concrete chemical structure and the way how these macromolecules arrange into higher order structures. Given the enormous importance of single-stranded polymers we took the challenge to try to synthesize 'infinitely' extended, covalently constructed and structurally defined, periodic 2D polymers and to explore their property space which is believed to be full of exciting surprises. ${ }^{[8]}$ In light of the recent discoveries around graphene such a research target has gained an even higher fascination. Not only would the development of reliable and robust synthetic routes leading to structurally rigorously proven representatives of molecular sheets be a major breakthrough for synthesis and chemistry; it also would open fascinating structural, analytical, application and theoretical aspects and options with an almost unimaginable impact on the natural sciences and technology.

This article draws a brief state-of-theart picture of these three selected cases to share the excitement of the enormous potential of modern polymer synthesis with the reader. It also emphasizes the role of organic chemistry whenever the realization of novel polymer structures is concerned and with this contrasts the presently sometimes encountered view that "everything can be done with the existing monomers and polymers" just by proper engineering and improved process technologies. The title reflects the three topics in that most of the polyarylenes are rigid-rod polymers, dendronized polymers are thick (up to diameters of 7-8 nm) and the targeted 2D polymers would be laterally infinite.

\section{Results and Discussion}

\subsection{Polyarylenes by Suzuki Polycondensation}

Suzuki polycondensation was discovered in the late 1980s at the Max-PlanckInstitute for Polymer Research in the main author's group and with the help of Prof. Gerhard Wegner together with the former doctoral student Matthias Rehahn and the support of Prof. W. James (Jim) Feast, Durham, U.K. ${ }^{[9]}$ Rehahn retrieved from the literature the original work by Suzuki and Miyaura on a Pd-mediated method for C-C bond formation. [5] This reaction requires the simultaneous presence of an aromatic boronic acid and an aryl halide to give the corresponding biphenyl derivative in extremely high yields. He also tried it out in a model reaction which went so well that it was obvious to try the same reaction for polymerization purposes, for which the reported (and confirmed) extremely high conversions per bond-formation step were an essential prerequisite for bringing about high molar mass products. Scheme 1 shows the first case of a polymerization using this coupling reaction which was later referred to as Suzuki polycondensation (SPC). Though at that time the molar masses were not yet satisfying, ${ }^{[10]}$ this work initiated a world-wide development which has led to a whole new family of partially high molar mass polyarylenes, many of which are described in a recent review article, ${ }^{[11]}$ though almost every month another example appears in the literature. It is a remarkable facet of this research that it has even found industrial application. ${ }^{[12]}$ Merck's branch, formerly known as Covion, Hoechst, used and still uses SPC for the synthesis of polymers as luminescent parts in their organic light-emitting diods. Practically all polymers prepared by SPC are linear polyarylenes which are considered rigid rod polymers.

The most recent success story in this area, which hopefully will also have an impact on industry, is a manifestation of the group's move from Berlin to Zürich. Paul Smith from the polymer technology group of ETH's Department of Materials became interested in the findings of doctoral student Ramchandra Kandre that highly pure monomers $\mathbf{1 b}$ and $\mathbf{2}$ can be polymerized on a multigram scale to the corresponding poly(meta-para-phenylene) 3 (Scheme 2) with a respectable molar mass. ${ }^{[13]}$ Since this polymer was obtained in larger amounts, a fraction could be obtained by simple precipitation that had the following characteristics: quantity, $1 \mathrm{~g} ; M_{\mathrm{w}}=255,000 \mathrm{~g} / \mathrm{mol}$; $P_{\mathrm{w}}=1140$ ! Despite this extremely high molar mass, highly resolved NMR spectra with narrow lines were obtained rendering the structure proof unequivocal. In Smith's laboratory the mechanical properties of this material were investigated. Films were found to be flexible and tough and could be deformed well beyond the yield point. Fig. 1 shows the results of tensile deformation tests (film thickness approx. $150 \mu \mathrm{m}$ ) with this polymer and, for comparison purposes, curves obtained with atactic polystyrene (PS), poly(methylmethacrylate) (PMMA), and polycarbonate (PC). Although the films of polymer $\mathbf{3}$ showed a somewhat lower stiffness, their macroscopic elongation at break surpassed those of the highly brittle PS and PMMA and approached that of PC, which is an engineering plastic reputed for its toughness. [14]

Stimulated by this exciting finding the ball has now been sent back to synthesis to pursue the new main goals of

i) creating a family of polymers grouped around polymer $\mathbf{3}$ which acts as a lead structure,

ii) reducing the catalyst amount to the absolute minimum, and

iii) investigating the polymerization mechanism.

Commonly SPC is considered a stepgrowth process. Tasks i and ii are presently under investigation whereby it should be mentioned that the reduction of Pd catalyst is not so much a matter of costs but rather of product properties. In melt processing high temperatures are applied and traces of residual $\mathrm{Pd}$ caused the material to turn dark. Ramchandra's most recent finding is that the initially used amount of catalyst can be reduced from $0.3-0.6 \%$ all the way to $0.03 \%$ while keeping the molar masses practically constant. This way, Pd residues in good samples can be as low as 2-3 ppm as determined by Laser Ablation Inductively Coupled Plasma Mass Spectrometry (LAICP-MS) for trace element analysis. With 


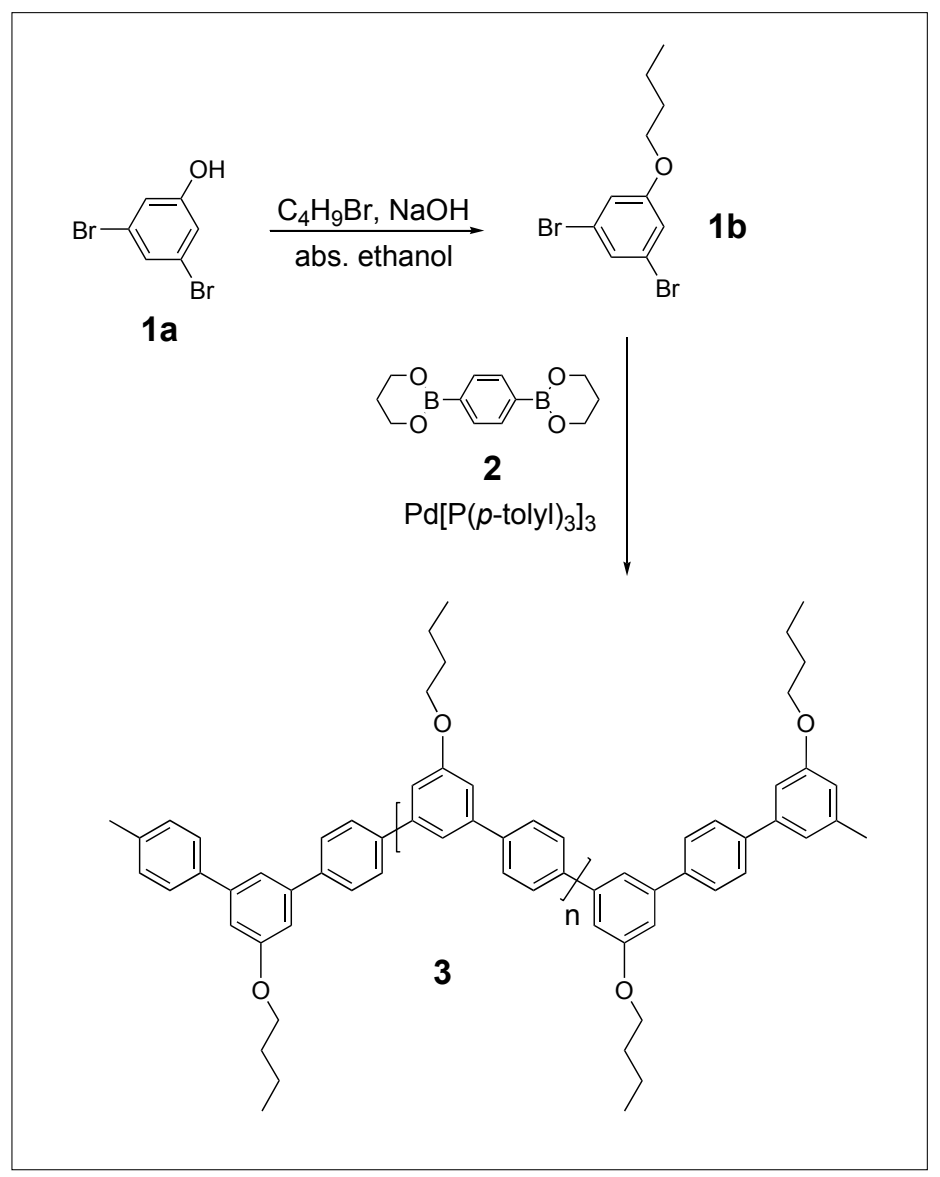

Scheme 2. Synthesis scheme for high molar mass poly(meta-paraphenylene) 3 with butoxy side chains using SPC

such low catalyst loadings and therefore very low metal residues color changes upon melt processing are no longer observed. Regarding the polymerization mechanism (aspect iii) it is a striking fact that the discussed fraction has an extremely high molar mass $\left(P_{\mathrm{w}}=1140\right)$, presumably too high for a step-growth process, despite the fact that it refers to a fractionated and, thus, nonrepresentative material. Can such values be obtained by step-growth procedures or does chain-growth play a role? Such considerations were already made some twenty years back in Mainz but were never followed experimentally (by us) and it was suspected that this was more likely to be the case for polymerizations using $\mathrm{AB}$ - rather than $\mathrm{AA} /$ $\mathrm{BB}$-monomers. There is recent precedence for so-called chain-growth polycondensations ${ }^{[15]}$ which may re-vitalize such thoughts and initiate research in this direction, specifically in light of the maturity and proven potential SPC has gained meanwhile.

A final aspect should be touched upon where research on SPC could additionally be directed at. Assume a polymer like $\mathbf{3}$ is equipped with substituents that make it fold up into a helical conformation: Such a helix would, regarding end-to end-distance, by far overtake everything that is known in the presently vigorously researched area of 'foldamers'. ${ }^{[16]}$ Whereas typical foldamers are in the oligomeric regime and therefore

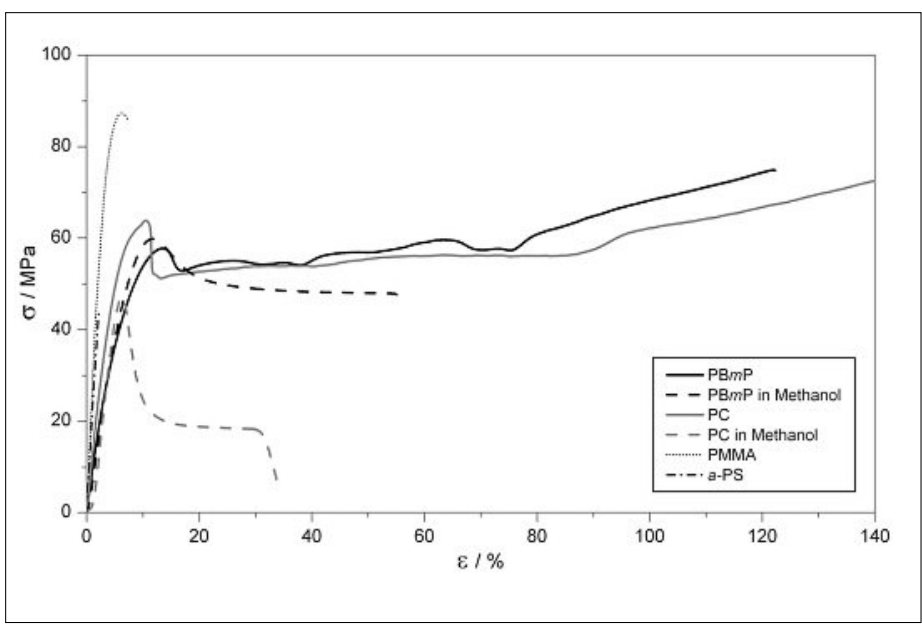

Fig. 1. Stress-strain curves, recorded at room temperature of meltcompression molded films of a fraction of polymer 3 (here referred to as $\mathrm{PBmP}$ ) (see text). For the structure of polymer $\mathbf{3}$, see Scheme 2. For reference purposes, corresponding curves of atactic-PS, PMMA, and $\mathrm{PC}$ are also shown, illustrating the excellent mechanical properties of the new polyarylene. Also plotted are stress-strain curves of polymer $\mathbf{3}$ and PC samples recorded while immersed in methanol, showing a superior resistance of the former polymer against environmental stress-cracking induced by this liquid. Reproduced from ref. [13] with permission by Wiley$\mathrm{VCH}$.

polymers in that they carry a sterically demanding, regularly branched substituent (dendron) at every repeat unit. ${ }^{[7]}$ This forces their backbone to unfold and stretch as the cartoon representation in Fig. 3 illustrates, and is the key reason for the many peculiarities that were encountered over the years with these polymers (Fig. 4). The molar mass of the dendrons may be as high as $10,000 \mathrm{~g} / \mathrm{mol}$ which instantaneously poses the question of how one has to tackle the synthesis. The answer is surprisingly simple: Choose a monomer concentration that is as high as possible so as to compensate as far as possible for the slowing down of propagation caused by both the shielding of both polymerizable unit and growing chain end and the low concentration of the former. If this nevertheless does not lead to formation of long chain dendronized polymers, as has been observed, e.g. for monomers with fourth generation dendrons (Fig. 5), ${ }^{[23]}$ an alternative strategy is available. This involves synthesis of a polymer with lower generation dendrons first, to which the missing generations are attached in a subsequent synthetic step. ${ }^{[24]}$

A large variety of dendronized polymers was prepared by us ${ }^{[7]}$ and others ${ }^{[7,25]}$ and their rather unusual properties investigated. Fig. 3 briefly illustrates a few of these properties. In one way or another, they are all related to rigidity, thickness, and the enormous number of functional groups in the periphery. For example, the formation of ordered supramolecular complexes between positively charged dendronized polymers and $d s$-DNA in which the DNA winds itself helically around the dendronized

\subsection{Dendronized Polymers: Multifunctional, Versatile Nanocylinders}

As mentioned in the introduction, dendronized polymers differ from other linear 


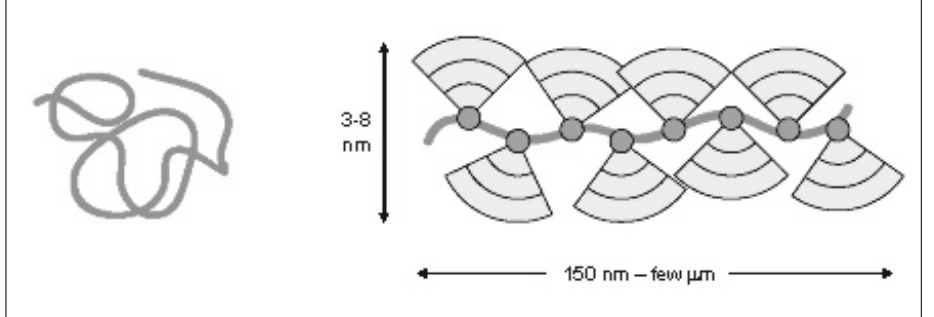

Fig. 3. Upon addition of spatially demanding dendrons (wedges) the randomly coiled backbone is forced to unfold and stretch, rendering the macromolecules rigid and to attain the shape of a cylinder with diameters in the range of 3-8 nm and lengths which may be between $100 \mathrm{~nm}$ and a few $\mu \mathrm{m}$, depending on the polymerization method.

polymer is not a common encounter. If positively and negatively charged conventional polyelectrolytes are mixed, they tend to collapse to an irregular globular material. Obviously, the thickness combined with the intrinsic rigidity of dendronized polymers and a proper charge balance are responsible for this astounding finding. Rather amazing is also the observation that high generation dendronized polymers can be considered as glassy materials already on the level of individual molecules. How else could one explain that a polymer can be dragged across a solid substrate (HOPG) with the SFM tip for hundreds of nanometers without suffering any conformational the creation of responsive representatives of this class of polymers as well as on the change! Presently research is focused on

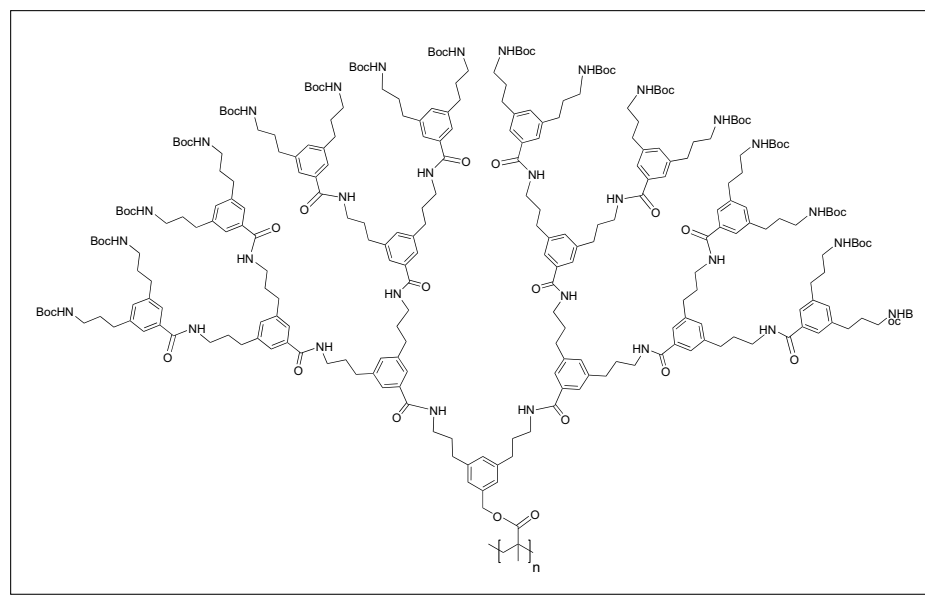

Fig. 5. A working horse fourth generation (G4) dendronized polymer with Boc-protected peripheral amine groups which can easily be converted into positively and, with a little more effort, also negatively charged groups rendering the corresponding polymers novel water-soluble polyelectrolytes general question whether these polymers' tunable thickness represents a new variable of polymer science very much like chain length and widths of distribution. The latter two variables have considerable impact on properties and one may wonder whether thickness could also have such a role.

But first let us address the issue of responsiveness. Recently, polymer PG2 was synthesized (Fig. 6).[26] It carries a triply branched second generation $(\mathrm{G} 2)$ dendron which is built from oligoethyleneoxy (OEO)

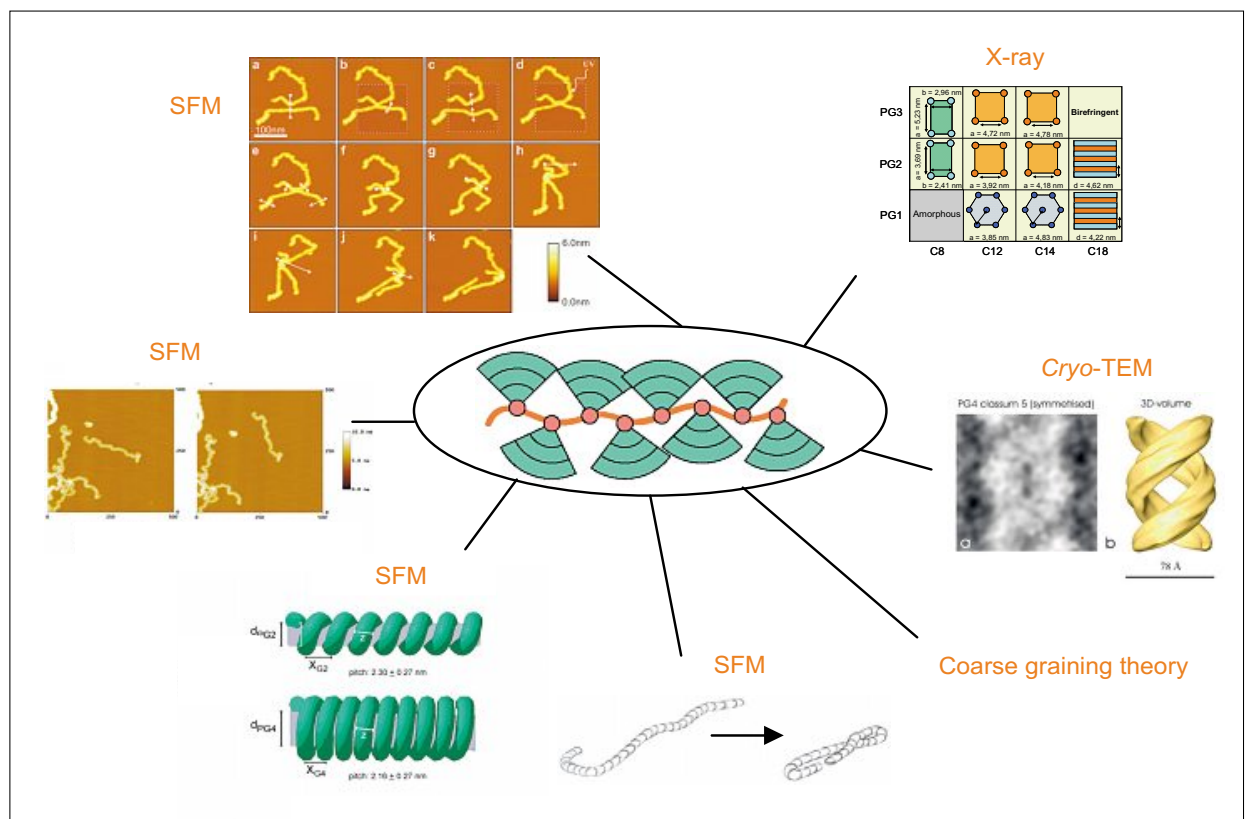

Fig. 4. Dendronized polymers shown in the center have several unprecedented properties and potential applications, some of which are illustrated here and will be explained in a counter clockwise fashion starting in the top left corner: single molecule experiments using the scanning force microscope (SFM), ${ }^{[17]}$ single molecule glasses as proven by SFM dragging experiments, ${ }^{[18]}$ creation of defined aggregates with biomacromolecules (here: ds-DNA helically wound around a positively charged dendronized polymer), ${ }^{[19]}$ spontaneous backfolding into duplex structures upon charging and when present in low concentration, ${ }^{[20]}$ formation of double-helical aggregates when positively charged and present in high concentration, ${ }^{[21]}$ and formation of defined 3D structures in bulk upon addition of surfactants to charged dendronized polymers. ${ }^{[22]}$ Finally, coarse-graining theoretical methods provide insight into why certain aggregates form. and benzene units. Also, - and this is important to note - it carries methoxy groups at the periphery. OEO and related oligomers/polymers are thermoresponsive which means that they are soluble in aqueous medium up to a certain temperature at which they then contract, dehydrate and collapse into aggregates large and compact enough to scatter light. This transition manifests itself therefore by the reversible change of a completely transparent solution to a turbid suspension. Such transitions can be used for sensing and other purposes. Having polymer PG2 at hand it was a natural choice to test whether it shows this kind of behavior. Much to our surprise it does in fact exhibit this property as is illustrated by the turbidity measurement shown in Fig. 7. Compared to literature data, ${ }^{[27]}$ this transition is unusually sharp, with an extremely small hysteresis, and also very fast. We therefore initiated a research program to explore this aspect deeper by taking PG2 as kind of a lead structure as one does it in pharmaceutical chemistry once an active compound has been discovered.

The second aspect which presently is of considerable interest is the thickness of dendronized polymers and whether this unique feature has any special meaning for polymer chemistry (like chain length and chain length distribution). For this purpose homologous series of polymers were synthesized which differed in dendron generation (thickness) but not in chain length. These series had either 800 or 6000 repeat units. ${ }^{[28]}$ They are presently under investigation regarding properties such as glass transition temperature, persistence length in the neutral and charged form, solubility, intrinsic viscosity, and compressibility in order to explore whether there is any correlation between properties and dendron generation. This work is being supported from the theoretical side through cooperation. Fig. 


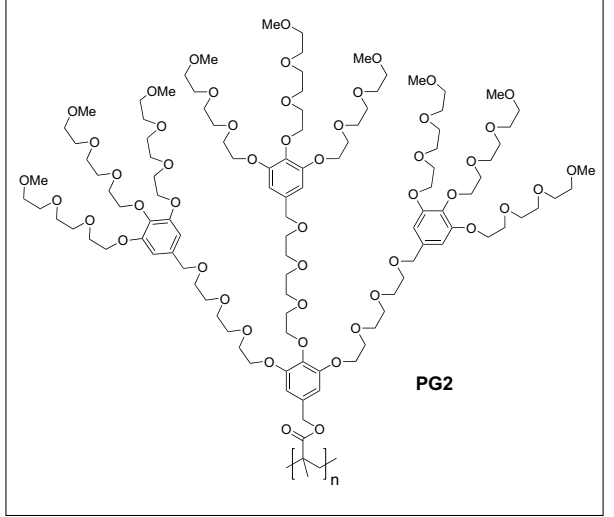

Fig. 6. Dendronized polymer PG2, a possible lead structure for thermoresponsive polymers with a very sharp, reversible, and fast transition

8 shows an unpublished so-called 'heavyduty' route to the first two members of such a homologous series.

\subsection{A Dream: Synthesis and Structure Analysis of Molecular Sheets}

It has been a dream of chemists for decades to try to create sheet-like (n-stranded) polymers whose structure meets the following criteria: 'infinitely' extended, covalently constructed, structurally defined, periodic (which means with long range order). This collection of criteria is what constitutes a $2 \mathrm{D}$ polymer ${ }^{[8]}$ and a few years back we set out to design and realize solutions which eventually will allow for both its synthetic realization and structural analysis. The mastering of the very high level of structure control inherently associated with 2D polymers can be considered a major driving force for our work in this area. Also, given the structural novelty of such unprecedented polymers, the exploration of their property space is another major motivation. Though enormous progress has been made, even today there is no man-made polymer known which meets the above criteria. The recent discoveries around graphene, which is a natural 2D polymer, ${ }^{[29]}$ has put a bright spotlight on this synthetic activity and we decided to provide a few insights on our recent, still unpublished work in this area here. ${ }^{[30]}$

One of the projects that are presently being pursued uses amphiphilic monomers at the air/water interface. This approach has the advantage that the monomers are already confined to two dimensions before they are connected. Though this pre-alignment at the interface may in the end not be a mandatory prerequisite for achieving $2 \mathrm{D}$ polymers, it is believed to be a helpful measure. A monomer used for the spreading and polymerization experiments is the calix[4]arene 4 (Fig. 9) which carries four anthracene units. The photochemically in-

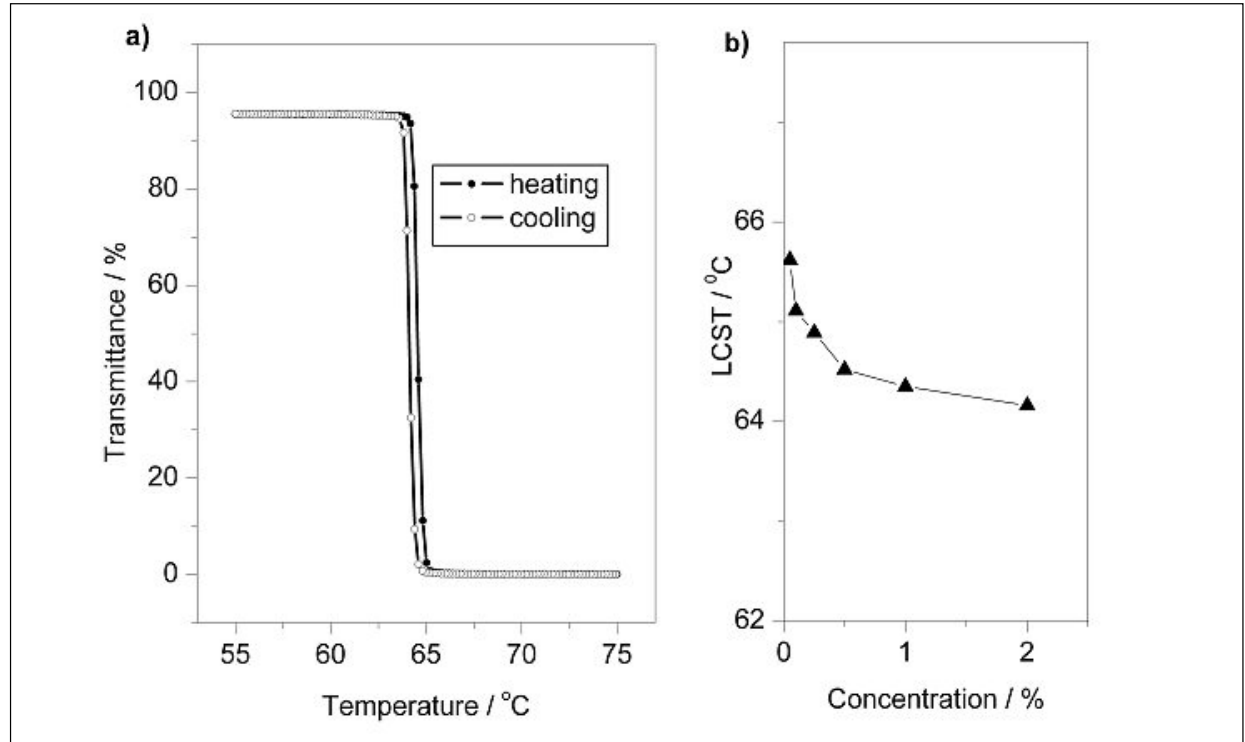

Fig. 7. Plots of transmittance vs temperature for 0.25 wt\% aqueous solutions of PG2 (a) and the concentration dependency of the transition temperature (b). Heating and cooling rate: $0.2^{\circ} \mathrm{C} / \mathrm{min}$.

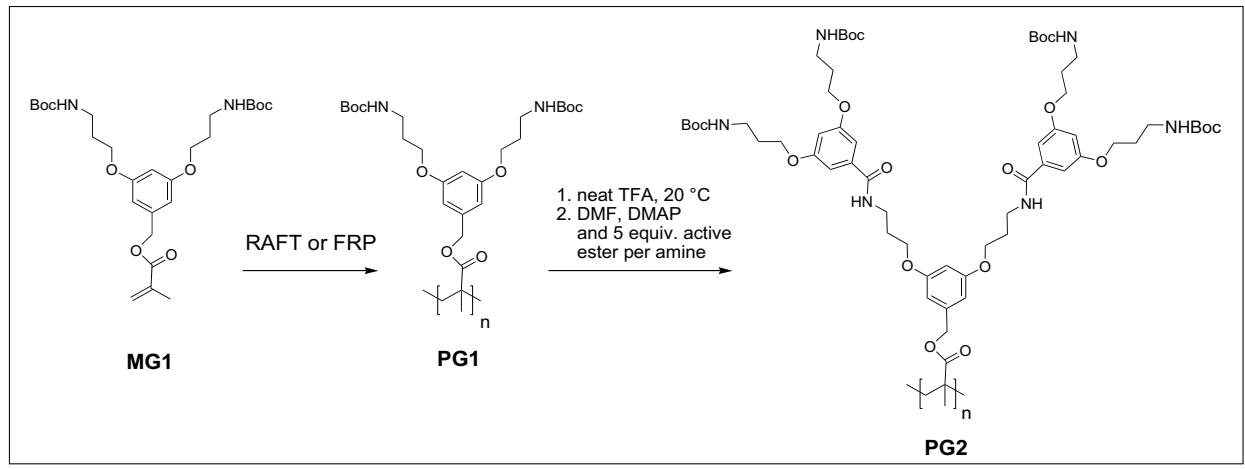

Fig. 8. The 'heavy-duty' route to the first members, PG1 and PG2, of a homologous series which also includes PG3 and PG4 (not shown). All polymers have the same chain length and chain length distribution to systematically explore whether their properties depend on dendron generation in any systematic way.

duced dimerization of such units has been well-studied and is used as the key reaction ideally resulting in the targeted lateral polymerizations. This concrete monomer served to prove the principle feasibility of polymerization at the interface with anthracene units but is not believed to be the optimum molecular structure for achieving

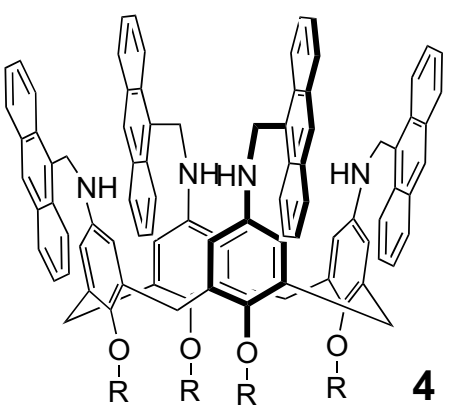

Fig. 9. Structure of the calix[4]arene-based amphiphilic monomer 4 which can be spread at the air/water interface to give a reversible monolayer. $\mathrm{R}=\left(\mathrm{CH}_{2} \mathrm{CH}_{2} \mathrm{O}\right)_{4} \mathrm{CH}_{3}$ a 2D polymer with undisturbed long-range order.

Upon photochemical treatment with UV light the film changed considerably. For example, if the untreated, compressed monolayer was mechanically challenged by macroscopically tearing it with a needle, the 'wounds' generated healed within seconds, as was monitored by Brewster angle microscopy (BAM). If however the film was irradiated prior to this treatment the cuts remained permanently visible (Fig. 10) even if the barrier was moved away.

The photochemically treated films could also be transferred to solid substrates to determine their thickness by ellipsometry (which confirmed that they are monolayers) and contact angle measurements (which supported the assumption that the crosslinked anthracenes are presented on top). Finally it was possible to also transfer them onto $\mathrm{Cu}$-grids normally used for electron microscopy. These grids had holes sized $45 \times 45 \mu \mathrm{m}$. Fig. 11 shows a film actually spanning some of the holes without being 


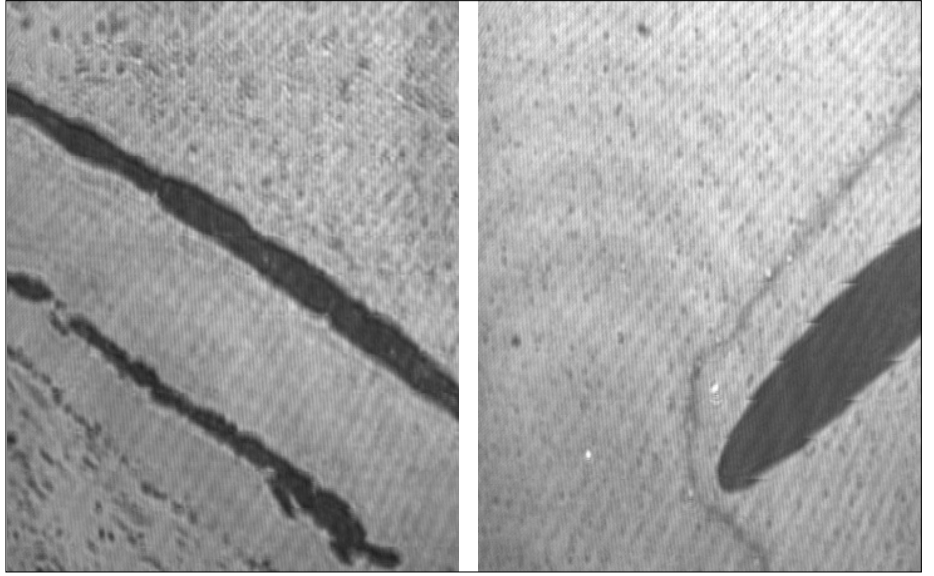

Fig. 10. BAM images of a photochemically treated, compressed monolayer of $\mathbf{4}$ after scratching with a needle. The scratches produced remain 'forever' even if the film is decompressed proving that a covalent cross-linking has taken place. The bottom length of these images is approximately $420 \mu \mathrm{m}$.

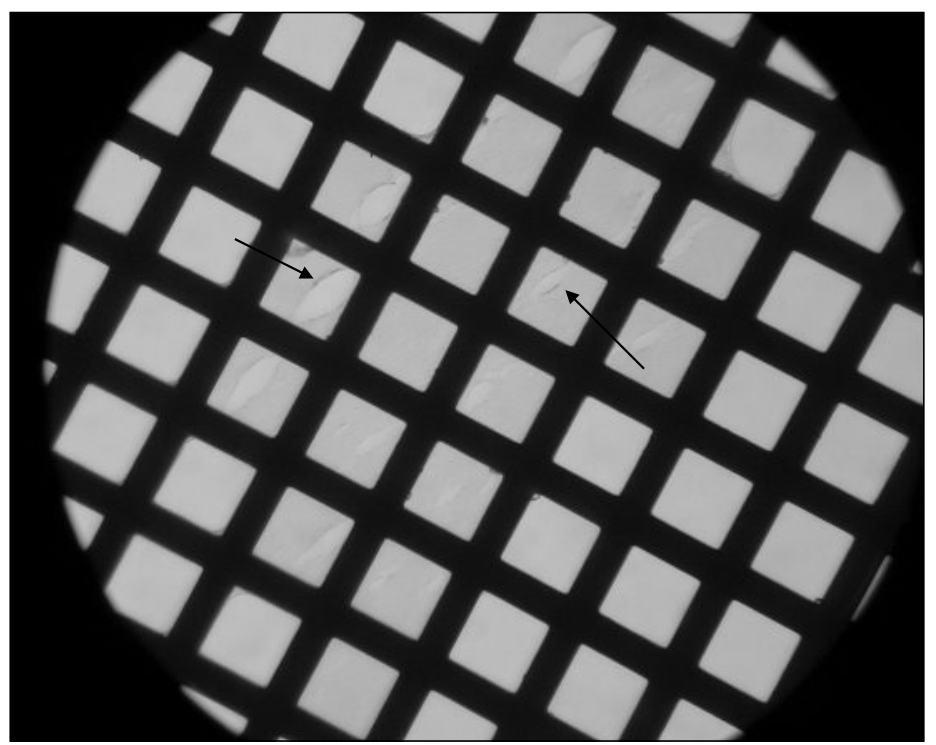

Fig. 11. Light microscopy image of an irradiated film of a monolayer of 4 produced at the air/water interface after its horizontal transfer on an electron microscopy grid with $2025 \mu \mathrm{m}^{2}$ sized holes. Some of the holes are spanned completely, on others the film is ruptured due to too high mechanical stress most likely imposed during transfer and drying. The arrows indicate cracks in the film which most likely form during transfer ation that industry has started to explore the technological potential of this class of polymers. As already mentioned for SPC, organic chemistry played an enormous role also here. Protection groups, peptide synthesis, hydroboration and many other tools of this discipline helped to lay the foundation for this success story. Finally, organic chemistry helped us pave the way to where we believe to be, namely at the edge of providing passable routes to $2 \mathrm{D}$ polymers matching the strict definition given above. Monomers need to be carefully designed and synthesized so as to cope with the numerous, seemingly insurmountable problems on the way to these laterally infinite, one monomer unit thick sheets with an internal long-range order. Polymer chemistry is an exciting field in which organic synthesis is refined to its top level and shows its potential for high impact innovation. Not everything can be achieved by engineering already existing polymers and optimizing process technologies!

\section{Acknowledgements}

We cordially thank the numerous coworkers whose excellent work, engagement, and creativity was the basis for the results reported here. Their names are referred to into the references. We would like to specifically mention Dr. Oleg Lukin, ETH Zürich, who helped us with the 2D project. It has always been a great pleasure to work with the following colleagues, who are the main collaboration partners for the projects mentioned: Profs. D. Günther, ETH Zürich, A. Halperin, CEA, Gernoble, W. Meier, Uni Basel, R. Mezzenga, Uni Fribourg, M. Kröger, ETH Zürich, H. C. Öttinger, ETH Zürich, J. P. Rabe, HU Berlin, M. Schmidt, Uni Mainz, P. Smith, ETHZürich. This work was generously supported by the Deutsche Forschungsgemeinschaft and Schweizer Nationalfonds as well as funds of the Max-Planck-Society, Freie Universität Berlin, and ETH Zürich, which is thankfully acknowledged.

Received: May 19, 2008 ruptured and others where the mechanical stress was obviously too high.

Though these few results look promising, the complication posed by the essential next step should not be underestimated: the structure analysis on the molecular level. Many ultrathin films have been reported over the years using random polymerization procedures ${ }^{[8]}$ and it is therefore essential to make every effort to prove that $2 \mathrm{D}$ polymers can truly be made, whose structural integrity meets that of any organic chemical.

\section{Summary and Outlook}

The three cases selected for this short review show that organic chemistry can have a profound impact on polymer synthesis. The Suzuki-Miyaura reaction is among the best chemical reactions known and not trying to use it for polymer synthesis would have been a 'sin of omission'. That SPC turned out to be a real success both in industrial and academic terms could not be foreseen, of course, but nicely underlines the enormous impact organic chemistry can have on materials' creation with an attractive property portfolio. Loading monomers up with huge substituents was in the beginning perhaps not accepted by everybody as being especially meaningful. Now that more than 30 research groups worldwide have added their valuable insights to the area of dendronized polymers, the potential of 'steric loading' has become much more visible and we find ourselves now in a situ-
[1] J. Okuda, R. Mülhaupt in 'Synthesis of Polymers', MST Series, Ed. A. D. Schlüter, Wiley-VCH, Weinheim, 1999, p. 123.

[2] a) W. A. Braunecker, K. Matyjaszewski, Adv. Polym. Sci. 2007, 32, 93; b) M. Save, Y. Guillaneuf, R. G. Gilbert, Aust. J. Chem. 2006, 59, 693.

[3] a) L. Brunsveld, B J B. Folmer, E. W. Meijer, R. P. Sijbesma, Chem. Rev. 2001, 101, 4071; b) A. W. Bosman, R. P. Sijbesma, E. W. Meijer, Materials Today 2004, 7, 34.

[4] a) P. Dziecok, S. S. Sheiko, K. Fischer, M. Schmidt, M. Möller, Angew. Chem., Int. $E d .1998,36,2812$; b) Y. Tsukahara, S. I. Namba, J. Iwasa, Y. Nakano, K. Kaeriyama, M. Takahashi, Macromolecules 2001, 34 , 2624; c) K. Fischer, M. Schmidt, Macromol. Rapid Commun. 2001, 22, 787; d) H. Shinoda, P. J. Miller, K. Matyjaszewski, 
Macromolecules 2001, 34, 3186; e) M. Zhang, T. Breiner, H. Mori, A. H. E. Müller, Polymer 2003, 44, 1449.

[5] N. Miyaura, T. Yanagi, A. Suzuki, Synth. Commun. 1981, 11, 513.

[6] A. D. Schlüter, G. Wegner, Acta Polym. 1993, 44, 59.

[7] a) A. D. Schlüter, J. P. Rabe, Angew. Chem., Int. Ed. 2000, 39, 864; b) A. Zhang, Prog. Chem. 2005, 17, 157; c) H. Frauenrath, Prog. Polym. Sci. 2005, 30, 325 ; d) A. D. Schlüter, Top. Curr. Chem. 2005, 245, 151.

[8] J. Sakamoto, J. van Heijst, O. Lukin, A. D. Schlüter, Angew. Chem., Int. Ed., review accepted.

[9] M. Rehahn, A. D. Schlüter, G. Wegner, W. J. Feast, Polymer 1989, 30, 1060.

[10] a) M. Rehahn, A. D. Schlüter, G. Wegner, Makromol. Chem. 1990, 191, 1991; b) M. Rehahn, A. D. Schlüter, G. Wegner, Makromol. Chem., Rapid Commun. 1990, $11,535$.

[11] A. D. Schlüter, J. Polym. Sci. Part A 2001, 39, 1533.

[12] a) K. Treacher, P. Stössel, H. Spreitzer, H. Becker, A. Falcou, PCT, WO 2003/048225 A2, to Covion Organic Semiconductors, Frankfurt, Germany; b) K. Brunner, M. M. de Kok-van Breemen, B. Langeveld, N. M. M. Kiggen, J. J. A. M. Bastiansen, J. W. Hofstraat, H. F. Boerner, H. F. M. Schoo, PCT WO 2004/072205 A2, to Koninklijke Philips Electronics, Eindhoven, Netherlands.

[13] R. Kandre, K. Feldman, H. E. H. Meijer, P. Smith, A. D. Schlüter, Angew. Chem., Int. Ed. 2007, 46, 4956.

[14] Polymer Handbook, 4th ed., Eds: J. Brandrup, E. H. Immergut, E. A. Grulke, Wiley, New York, 1999.

[15] B. Voit, Angew. Chem., Int. Ed. 2000, 39, 3407.
[16] D. J. Hill, M. J. Mio, R. B. Prince, T. S. Hughes, J. S. Moore, Chem. Rev. 2001, 101, 3893.

[17] a) J. Barner, F. Mallwitz, L. Shu, A. D. Schlüter, J. P. Rabe, Angew. Chem., Int. Ed. 2003, 42, 1932; b) R. Al-Hellani, J. Barner, J. P. Rabe, A. D. Schlüter, Chem. Eur. J. 2006, 12, 6440.

[18] a) C. Ecker, N. Severin, L. Shu, A. D. Schlüter, J. P. Rabe, Macromolecules 2004, 37, 2484; b) J. J. Das, M. Yoshida, Z. A. Fresco, T.-L. Choi, J. M. J. Fréchet, A. K. Chakraborti, J. Phys. Chem. B 2005 , 109, 6535 .

[19] I. Gössl, L. Shu, A. D. Schlüter, J. P. Rabe, J. Am. Chem. Soc. 2002, 124, 6860.

[20] W. Zhuang, E. Kasemi, Y. Ding, M. Kröger, A. D. Schlüter, J. P. Rabe, $A d v$. Mater. 2008, 20, 3204.

[21] C. Böttcher, B. Schade, C. Ecker, J. P. Rabe, L. Shu, A. D. Schlüter, Chem. Eur J. 2005, 11, 2923.

[22] a) N. Canilho, E. Kasemi, R. Mezzenga, A. D. Schlüter, J. Am. Chem. Soc. 2006, 128, 13998; b) N. Canilho, E. Kasemi, A. D. Schlüter, R. Mezzenga, Macromolecules 2007, 40, 2822; c) N. Canilho, E. Kasemi, A D. Schlüter, J. Ruokolainen, R. Mezzenga, Macromolecules 2007, 40, 7609.

[23] A. Zhang, B. Zhang, E. Wächtersbach, M. Schmidt, A. D. Schlüter, Chem. Eur. J. 2003, 9, 6083 .

[24] A. Zhang, L. Shu, Z. Bo, A. D. Schlüter, Macromol. Chem. Phys. 2003, 204, 328.

[25] For a few recent examples not contained in ref. [7], see: a) W. Q. Shi, Y. H. Zhang, C. J. Li, Z. Wang, X. Zhang, Y. Zhang, Y. M. Chen, Polymer 2006, 47, 2499; b) V. Percec, J. G. Rudlick, M. Peterca, E. Aqad, M. R. Imam, P. A. Heiney, J. Polym. Sci. Part A, Polym. Chem. 2007, 45, 4974; c) S. Rajaram, T. L. Choi, M. Rolandi, J. M. J. Fréchet, J. Am. Chem. Soc. 2007
129, 9619; d) X. Q. Xiong, Y. M. Chen, S. Feng, W. Wang, Macromolecules 2007, 40, 9084; e) Z. A. Tan, R. P. Tang, E. J. Zhou, Y. J. He, C. H. Yang, F. Xi, Y. F. Li, J. Appl. Polym. Sci. 2008, 107, 514; f) X. Xiao, Y. G. Wu, M. H. Sun, J. J. Zhou, Z. S. Bo, L. Li, C. M. Chan, J. Polym. Sci. Part A, Polym. Chem. 2008, 46, 574.

[26] a) W. Li, A. Zhang, A. D. Schlüter, Macromolecules 2008, 41, 43; b) W. Li, A. Zhang, H. Wu, K. Feldmann, P. Walde, A. D. Schlüter, Macromolecules 2008, 41 , 3659; c) W. Li, A. Zhang, A. D. Schlüter, Chem. Commun. 2008, accepted.

[27] For example, see: a) X. Wang, X. Qiu, C. $\mathrm{Wu}$, Macromolecules 1998, 31, 2972; b) J.-F. Lutz, O. Akdemir, A. Hoth, J. Am. Chem. Soc. 2006, 128, 13046; c) J.-F Lutz, A. Hoth, Macromolecules 2006, 39, 893.

[28] Y. Guo, A. Zhang, A. D. Schlüter, in preparation.

[29] a) K. S. Novoselov, A. K. Geim, S. V. Morozov, D. Jiang, Y. Zhang, S. V. Dubonos, I. V. Grigorieva, A. A. Firsov, Science 2004, 306, 666; b) E. J. Duplock, M. Sheffler, P. J. D. Lindan, Phys. Rev. Lett. 2004, 92, 225502; c) K. S. Novoselov, D. Jiang, F. Schedin, T. J. Booth, V. V. Khokevich, S. V. Morozov, A. K. Geim, Proc. Natl. Acad. Sci. USA 2005, 102, 10451; d) Y. Zhang, Y. W. Tan, H. L. Stormer, P. Kim, Nature 2005, 438 , 201; e) Y. Zhang, J. P. Small, M. E. S. Amori, P. Kim, Phys. Rev. Lett. 2005, 94 , 176803; f) A. K. Geim, K. S. Novoselov, Nature Mater. 2007, 6, 183.

[30] C. Münzenberg, A. Rossi, K. Feldman, R. Fiolka, A. Stemmer, K. Kita-Tokarczyk, W. Meier, O. Lukin, A. D. Schlüter, Chem. Eur. J., accepted. 